\title{
Conceptual Information Systems Discussed through an IT-Security Tool
}

\author{
Klaus Becker ${ }^{1}$, Gerd Stumme ${ }^{2}$, Rudolf Wille ${ }^{2}$, Uta Wille ${ }^{3}$, Monika Zickwolff ${ }^{4}$ \\ 1 Entrust Technologies (Switzerland) Ltd liab. Co, Glatt Tower, \\ CH-8301 Glattzentrum, Switzerland; klaus.becker@entrust.com \\ 2 Technische Universität Darmstadt, Fachbereich Mathematik, D-64289 \\ Darmstadt, Germany; \{stumme, wille\}@mathematik.tu-darmstadt.de \\ 3 Jelmoli AG, Information Systems, Postfach 3020, \\ Ch-8021 Zürich, Switzerland; wille_u@jelmoli.ch \\ 4 NaviCon Gesellschaft für Begriffliche Wissensverarbeitung mbH, \\ Heinrichstrasse 9, 60327 Frankfurt/Main, Germany; zickwolff@navicon.de
}

\begin{abstract}
Conceptual Information Systems are based on a formalization of the concept of 'concept' as it is discussed in traditional philosophical logic. This formalization supports a human-centered approach to the development of Information Systems. We discuss this approach by means of an implemented Conceptual Information System for supporting IT security management in companies and organizations.
\end{abstract}

\section{Contents}

1. Conceptual Information Systems

2. IT-Security Management

3. Formal Concept Analysis and TOSCANA

4. A Conceptual Information System for IT-Security Management

5. Further Developments

\section{Conceptual Information Systems}

Information Systems are understood as systems for input, storage, association, processing, analysis, retrieval, and output of information which are designed by organic, technical and organisational principles to support learning and communication processes. Most information systems have the purpose to assist human beings in creating knowledge. Since human knowledge heavily depends on common sense, preknowledge, context and culture, the development of those systems should not only follow a technical understanding of information and knowledge, but also a human-related understanding including meanings, interpretations, purposes etc. Ron Brachman and others have therefore advocated a human-centered approach that supports asking, exploring, analyzing, interpreting, and learning in interaction with the information system (cf. [5], [4]). Their claims have been stated as system requirements listed in [25] which we recall with minor modifications: 
1. An information system should represent and present to the user the underlying domain in a natural and appropriate fashion. Objects from the domain should be easily incorporated into queries.

2. The domain representation should be extendible by addition of new categories formed from queries; these categories (and their representative individuals) must be usable in subsequent queries.

3. It should be easy to form tentative segmentations of data, to investigate the segments, and to re-segment quickly and easily; there should be a powerful repertoire of viewing and analysis methods, and these methods should be applicable to segments.

4. Users should be supported in recognizing and abstracting common analysis (segmenting and viewing) patterns; it must be easy to apply and modify these patterns.

5. There should be facilities for monitoring changes in classes or categories over time.

6. An information system should increase the transparency of the processes of analysis and retrieval and should document their different stages.

7. Analysis and retrieval tools should take advantage of explicitly represented background knowledge of domain experts, but should also activate the implicit knowledge of experts.

8. An information system should allow highly flexible processes of analysis and retrieval respecting the open and procedural nature of productive human thinking; this means in particular to support intersubjective communication and argumentation.

For fulfilling the listed requirements for a human-centered development of information systems, it is promotive to base the formal representation of human knowledge on a broad understanding of knowledge. According to the traditional philosophical logic, knowledge is grounded on the formation of concepts from which humans form their judgments (cf. [30]). Thus, concepts are essential for the creation and representation of knowledge. For human-centered developments of information systems, we therefore suggest to use a formalization of the philosophical understanding of concepts for the formal representation and presentation of knowledge; such a formalization is performed in Formal Concept Analysis [7]. We name human-centered information systems developed on the basis of Formal Concept Analysis "Conceptual Information Systems". In the following we discuss the conception of "Conceptual Information Systems" through an example of such system that has been developed to support the security management of information technology [2].

\section{IT-Security Management}

The task of Information Technology (IT) is to assure the availability, confidentiality, and integrity of the data of an organization. In the past few years the main focus has been on the availability. With an increasing exchange of data via 
local and public networks the protection of the data against loss of confidentiality and integrity has become a major issue. Reports on damages indicate that it is not sufficient to react on damages once they appear. The management of companies and public organizations needs a systematic support for an effective risk management, such that one can recognize threats well in advance and can prevent them by appropriate safeguards.

The past has shown that individual risk analysis is very costly and often results are not in a reasonable relation to the timely and financial expenses. Furthermore, a major part of the resulting safeguards is independent of the organization, for instance the protection of server or PCs by passwords. A solution is to codify the safeguards which are independent from the individual organization in an IT security management catalogue. Then only the particular aspects of an organization have to undergo an individual risk analysis. The implementation of this IT security catalogue provides an adequate security level for systems with medium-level protection requirements.

For a continuously high security level it is not sufficient to implement a onetime security concept. It is necessary to create processes assuring the continuous interplay of requirement analysis, realisation, and control. The requirement analysis determines the needed security level. It depends on the potential damage and on the value of the objects to be protected. In IT this means before all the value of the processed data. This value is determined by the used IT resource, the IT process, and the organizational unit which applies the IT resource in the particular IT process. For these IT units, the needed level of protection is determined in the requirement analysis. An example for an IT unit is the combination of the IT resource 'mailing system', the IT process 'operation', and the organizational unit 'data processing center'.

One of the most important tasks of IT security management is the realisation of the conception, i. e., the assignment of the requirements of the IT security catalogue to the organizational units, as well as the derivation of concrete directives and checklists for the organizational units from the requirements. An important success factor of this task is a high acceptance of the directives by the involved organizational units. This means primarily a suitable structuring with regard to the organizational units:

- Comparable matters of fact should result in comparable requirements (e.g., virus protection for $\mathrm{PCs}$ ).

- Each IT unit should have assigned to only its specific requirements, since the implementation of the whole catalogue without taking into account the specific security level needed is rarely reasonable. For instance, PCs in the management underly other requirements than PCs in the R\&D department.

- Requirements should be ordered by priority. For instance, the protection against breakdown of PCs is more important in production than in marketing departments.

A regular verification of the obtained security level is a core part of IT security management. This includes surveying the application of the directives as well as their adaption to changing requirements or structures. 


\section{Requirements to an IT security management tool}

Due to the large amount of combinations of IT units and threats, the requirement analysis reaches a high degree of complexity even for small and medium-sized organisations. For realizing the IT security conception, the requirements have to be assigned to the relevant organizational units in an effective and transparent way. The system must support the comparison of typical situations and assigned requirements. In the realisation phase, it must also allow the integration of existing directives and checklists. The verification of the obtained security level requires visualization of the obtained level for different classes of IT units, and support for uncovering inconsistencies. These operative tasks determine directly the requirements for the IT security management tool:

1. The tool has to provide a data model and means of its administration for the requirement analysis, i. e., the construction of IT units and the registration of threats, and for the realization, i. e., the assignment of requirements, directives, and checklists.

2. For revision, the underlying IT security management catalogue must be extendable or replacable.

3. For constitution and maintenance of the data, as well as for the analysis and control of the actual level of security, a graphical user interface is needed.

NaviCon Gesellschaft für Begriffliche Wissensverarbeitung mbH and $r^{3}$ security engineering ag have developed an IT security management tool to fit these needs. The tool is based on the management system TOSCANA for Conceptual Information Systems which has been developed at the Technische Universität Darmstadt. TOSCANA and its underlying mathematical theory, Formal Concept Analysis, are introduced in the next section. The IT security management tool is presented in Section 4 .

\section{Formal Concept Analysis and TOSCANA}

The philosophical understanding of a concept as a unit of thought constituted by its extension and its intension has been influential to the western culture from the seventeenth century until today (cf. [29]). For instance, the German Industrial Standards DIN 2330 "Begriffe und Benennungen" and DIN 2331 "Begriffsysteme und ihre Darstellung" are based on this understanding of concepts. Let us recall that the extension of a concept comprises all objects belonging to the concept while the intension (comprehension) grasps all attributes (properties, meanings) of those objects.

For allowing a mathematical description of extensions and intensions, Formal Concept Analysis always starts with a formal context defined as a triple $(G, M, I)$ where $G$ is a set of (formal) objects, $M$ is a set of (formal) attributes, and $I$ is a binary relation between $G$ and $M$ (i.e. $I \subseteq G \times M$ ); in general, $g \operatorname{Im}$ ( $\Leftrightarrow$ $(g, m) \in I)$ is read: "the object $g$ has the attribute $m$ " (cf. [7]). In Figure 1, a formal context (taken from [6]) is described by a table in which the crosses 


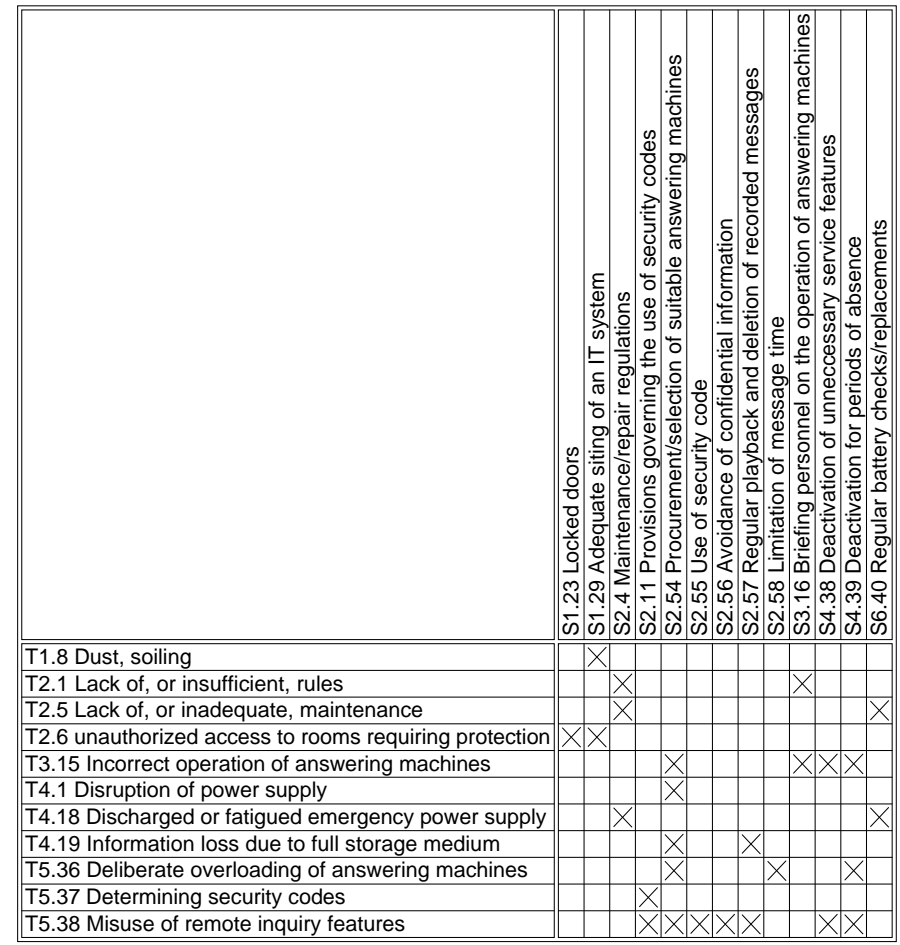

Fig. 1. A formal context concerning a telephone answering machine

represent the binary relation $I$ between the object set $G$ (comprising the threats concerning a telephone answering machine) and the attribute set $M$ (consisting of safeguards to prevent those threats). A formal concept of a formal context $(G, M, I)$ is defined as a pair $(A, B)$ with $A \subseteq G$ and $B \subseteq M$ such that $(A, B)$ is maximal with the property $A \times B \subseteq I$; the sets $A$ and $B$ are called the extent and the intent of the formal concept $(A, B)$. The subconcept-superconcept-relation is formalized by

$$
\left(A_{1}, B_{1}\right) \leq\left(A_{2}, B_{2}\right): \Longleftrightarrow A_{1} \subseteq A_{2} \quad\left(\Longleftrightarrow B_{1} \supseteq B_{2}\right) .
$$

The set of all formal concepts of a formal context $(G, M, I)$ together with the order relation $\leq$ is always a complete lattice, called the concept lattice of $(G, M, I)$ and denoted by $\underline{\mathfrak{B}}(G, M, I)$. Figure 2 visualizes the concept lattice by a (labeled) line diagram. In a line diagram of a concept lattice, the name of an object $g$ is always attached (from below) to the little circle representing the smallest concept with $g$ in its extent (denoted by $\gamma g$ ); dually, the name of an attribute $m$ is always attached (from above) to the little circle representing the largest concept with $m$ in its intent (denoted by $\mu m$ ). This labelling allows us to read the context relation from the diagram because $g I m \Longleftrightarrow \gamma g \leq \mu m$, in words: 


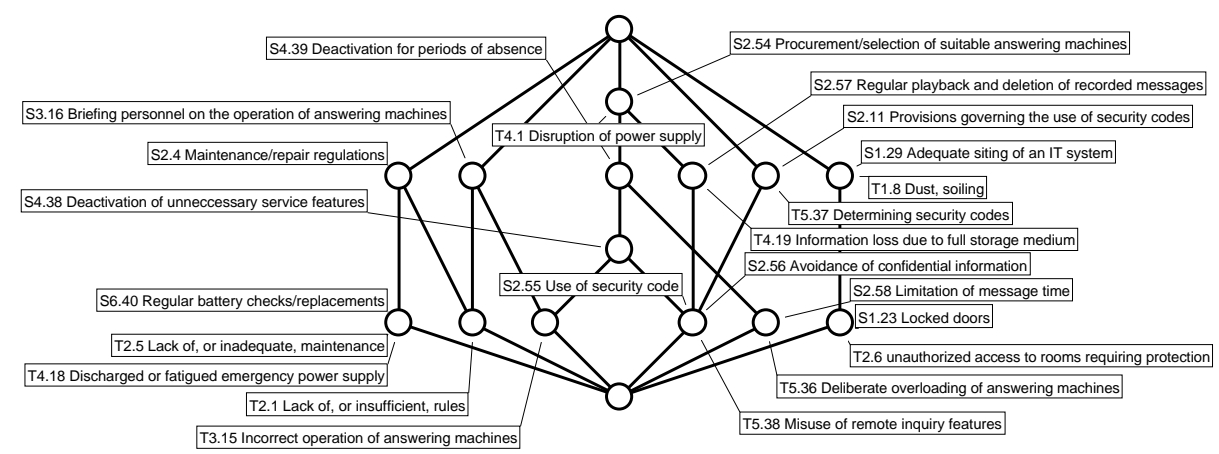

Fig. 2. The concept lattice of the context of Figure 1

the object $g$ has the attribute $m$ if and only if there is an ascending path from the circle representing $\gamma g$ to the circle representing $\gamma m$.

The extent and intent of each concept $(A, B)$ can also be recognized because

$$
A=\{g \in G \mid \gamma g \leq(A, B)\} \text { and } B=\{m \in M \mid(A, B) \leq \mu m\} .
$$

For example, the little circle in the line diagram of Figure 2 labelled with "S4.39 Deactivation of answering machines for periods of absence" represents the formal concept with the extent $\{$ "T3.15 Incorrect operation of answering machines", "T5.36 Deliberate overloading of answering machines", "T.5.38 misuse of remote inquiry features" $\}$ and the intent $\{$ "S2.54 Procurement/selection of suitable answering machines", "S.4.39 Deactivation of answering machines for periods of absence" .

Graphically represented concept lattices are useful in discovering and understanding conceptual relationships in given data. Therefore the development of "Conceptual Information Systems" may use concept lattices as query structures for databases. Such interplay of concept lattices and databases has already been designed in the theory of conceptual data systems. A conceptual data system consists of a (relational) database and a conceptual scheme. A conceptual scheme is a collection of formal contexts, called conceptual scales, together with line diagrams of their concept lattices; such systems are implemented with the management system TOSCANA (see [21],[13],[28]). For a chosen conceptual scale, TOSCANA presents a line diagram of the corresponding concept lattice indicating all objects stored in the database in their relationships to the attributes of the scale. For instance, the formal context in Figure 1 is an example of a conceptual scale taken from the data presented in the IT Baseline Protection Manual of the Bundesamt für Sicherheit in der Informationstechnik [6]. A TOSCANA system based on the data in [6] (as discussed in [22]) would show, after choosing that scale, the appertaining lattice diagram in Figure 2.

The power of a TOSCANA system lies in the possibility to refine a presented concept lattice by another one so that one obtains either a nested line diagram of a combination of both lattices (see Figure 5 in Section 4 ) or a line diagram of the 


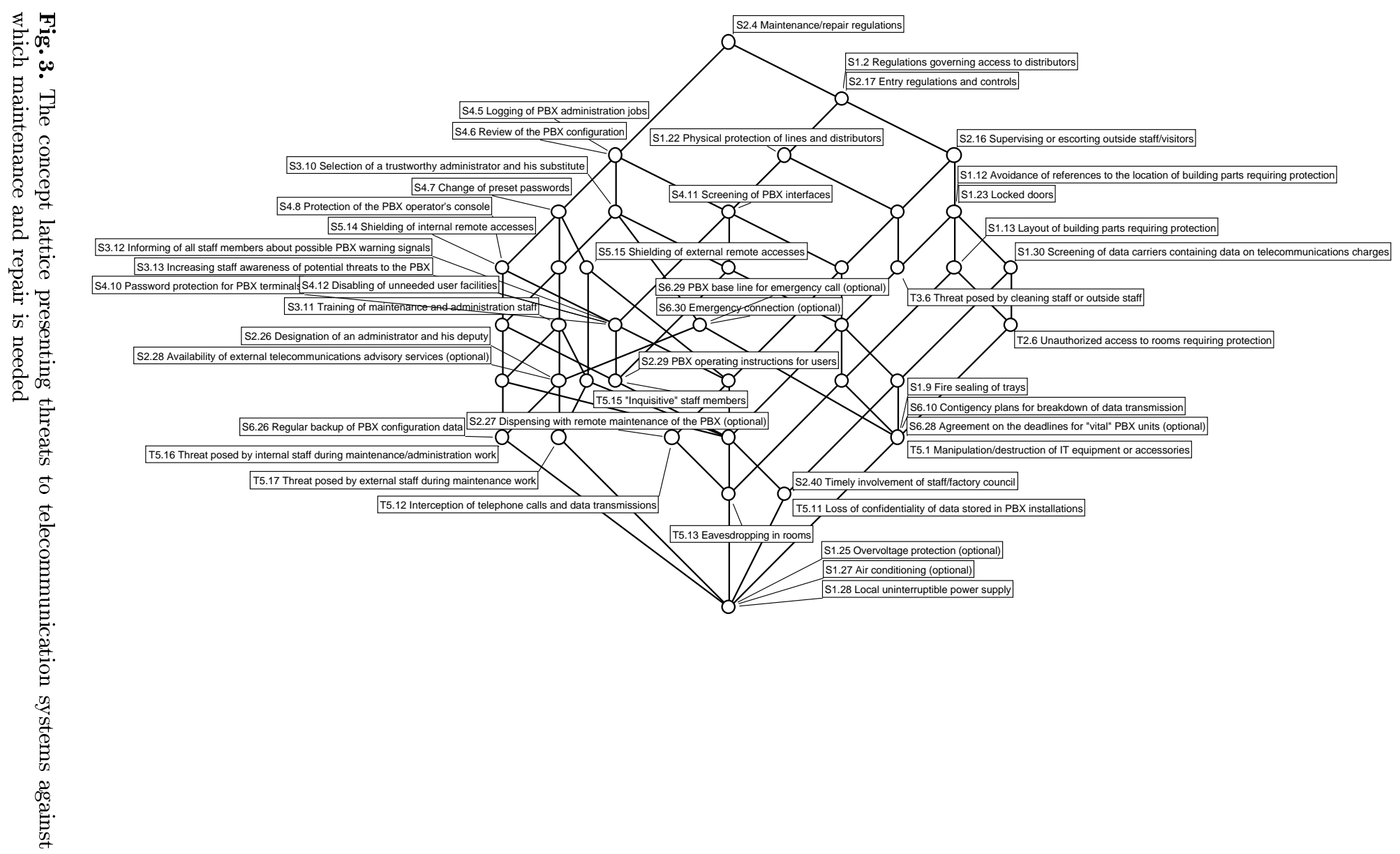


second refining a chosen concept of the first. If for instance the study of Figure 2 leads to the assignment of directives and checklists for maintenance and repair regulations (S2.4), it would be advisible to consider maintenance and repair of other facilities such as telecommunication systems; this can be supported by Figure 3 obtained by zooming into the node labelled by S2.4 with the conceptual scale "Telecommunication Systems (Private Branch Exchange)". The concept lattice in Figure 3 indicates how rich the combinations of necessary safeguards are for preventing threats to telecommunication systems against which maintenance and repair is needed. Our small example may give already an idea how conceptual information systems developed with TOSCANA allow highly flexible processes of analysis and retrieval in respecting the open and procedural nature of human thinking (cf. Section 1); these processes are not restricted because the zooming procedure potentially enables to navigate through the whole database (cf. [31]).

\section{A Conceptual Information System for IT-Security Management}

The IT Baseline Protection Manual of the Bundesamt für Sicherheit in der Informationstechnik provides safeguards against threats for generic components of an IT system. For the implementation of a security concept in an organization or company, the safeguards have to be transformed in more specific directives and checklists. Therefore, NaviCon Gesellschaft Für BeGriffliche WISSENSVERARBEITUNG MBH and $r^{3}$ security engineering ag have developed an IT security management tool which provides a higher level of detail. Its basic data model is given in Figure 4. The model consists of three major parts: The IT Security Management Catalogue comprises a generic company independent catalogue of threats and related safeguards, which can be extended by company specific facts.

IT Units represent the relationship between the company specific IT processes and the organizational units with regard to the used IT resources. The third part provides directives and checklists which are assigned to the IT units with respect to the requirements.

The data model is implemented in a relational database. Its implementation and maintenance is done via a Graphical User Interface which is based on ANACONDA, a preparation tool for Conceptual Information Systems. The data are analysed by the management system TOSCANA. It provides the visualization of relationships between the conceptual scales by nested line diagrams and allows an on-line navigation through the whole database. The conceptual scheme which is needed by TOSCANA is generated and updated by ANACONDA.

We describe how TOSCANA supports IT security analysis by means of an example. Figure 5 shows a nested line diagram which is composed by the three conceptual scales 'Existence of directives', 'Key Control' and 'Faults'. When we first have a look at the large left ellipse of the diagram, then we see that there are $1+3+4+2=10$ requirements for which there are no directives formulated at the moment ('Maßnahme nicht vorhanden' = directives not existent). Two of 


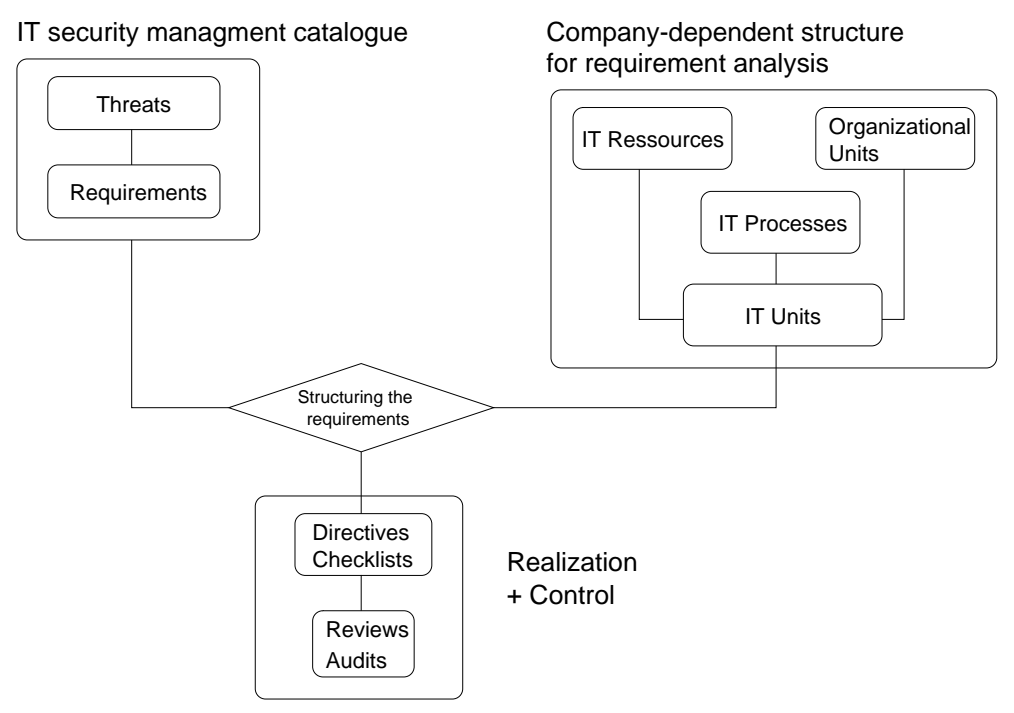

Fig. 4. The data model of the IT security management tool

them are key control, i. e., requirements which are considered as very important. Hence, for these two requirements, directives and checklists should be provided immediately, while the other requirements can be regarded later.

But also for key control requirements where directives are already present, one has to verify if they are appropriate. There are $1+1+2+9+2+3=18$ such requirements which can be found in the rightmost medium-sized ellipse which is determined by the attributes 'Maßnahme vorhanden' (= directives existent) and 'Key Control'. By drill-down into this ellipse, we obtain Figure 6. Here we can see in more detail how these requirements are distributed with regard to different types of faults. For instance, all requirements assigned to 'Human Resources' are related to 'Faults', 'Human failures' and 'Organizational Shortcomings'. Since we have zoomed into the rightmost ellipse of Figure 5 (Maßnahme vorhanden) we know that there are directives and checklists provided. By clicking on 'Maßnahme anzeigen' (= show directives), we can switch to the GUI based on ANACONDA where we obtain more information about these directives. The directives assigned to Human Resources include for instance regulations about security check procedures and immediate deleting of accounts when employees leave the company.

Concluding this section, we discuss to what extent the system requirements provided by Brachman et al. (cf. Section 1) are fulfilled by the IT security management tool.

1. The entities of the data model (threats, requirements, IT resources, organizational units, etc.) reflect the way IT security experts see their organization. By differentiating these entities in conceptual scales, they can be activated 


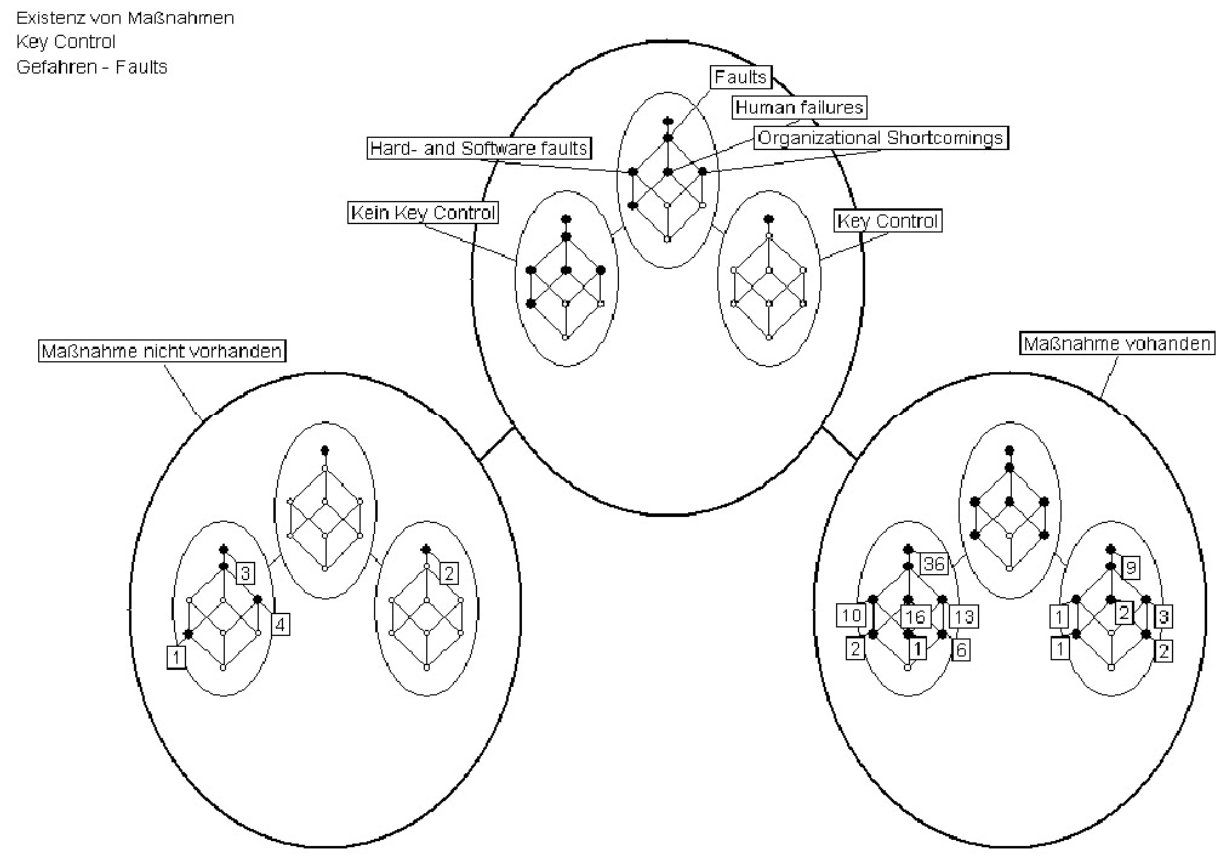

Fig. 5. Analysis of existing directives

in ad hoc queries. One remaining difficulty is that, in the present state, one entity type has to be chosen in advance as object type (in the formalization of Formal Concept Analysis). The extension of the theory in order to provide more flexibility in this aspect is in progress.

2. Categorical structures are described by conceptual scales which can be added to the system at any time. Even during the analysis process, new insights may give rise to further conceptual scales. In the case of the IT security tool, in order to create a new conceptual scale, it is sufficient to select the attributes describing these categories; the conceptual scale is then automatically derived from the present data by ANACONDA.

$3 .+4$. The possibility of combining arbitrary conceptual scales provides an almost unlimited multitude of conceptual segmentations and patterns which offer each different views on the data.

5. At the moment, monitoring changes over time is not implemented in the IT security management system. However, one could extend the data model by time stamps which indicate the moment of introducing new directives, and provide a conceptual scale covering this temporal aspect. This is implemented in other applications of Conceptual Information Systems, for instance in a system for monitoring an incineration plant ([9]). 


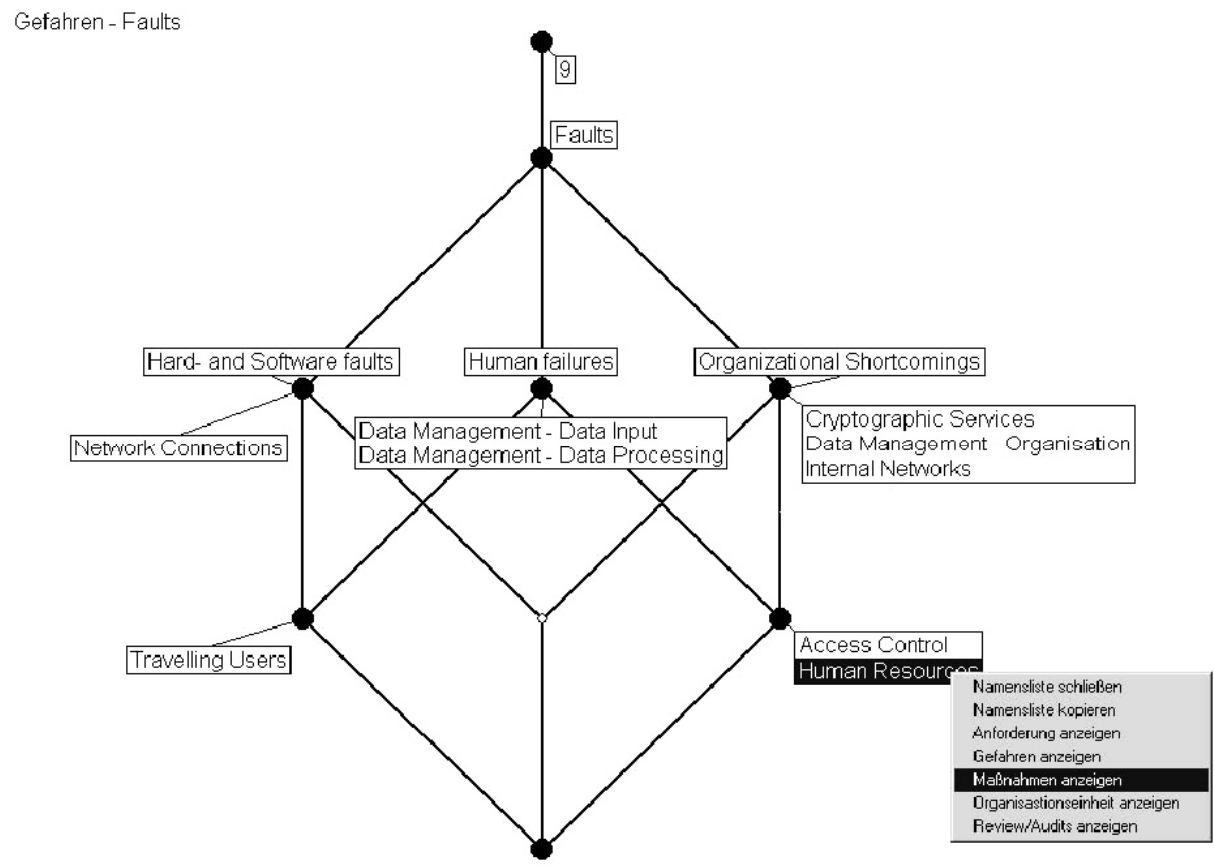

Fig. 6. Zooming into the rightmost concept of the nested line diagram in Figure 5

6. The transparency of the analysis process is asserted by the fact that aggregation is only performed by set union. Hence all information about the individual objects is obtainable at all time.

TOSCANA offers to the user a history drill- down. It lists the attributes to which the actual concept is restricted. A global history tracing the overall analysis process is not implemented at the moment.

7. Background knowledge of domain experts is represented in the structure of the conceptual scales in which the experts have explicitly coded formal aspects of their knowledge. The connection to implicit background knowledge is activated by the semantics of the attributes in the conceptual scales.

8. By visualizing the distribution of objects over related concepts, line diagrams are more appropriate than single numerical values (such as averages and standard deviations) to support the communication between experts and users.

Overall, Conceptual Information Systems offer conceptually shaped landscapes of structurally coded knowledge allowing diverse excursions, during which a learning process yields an increasingly better understanding of the domain (cf. [31]). The graphical representation of interesting parts of the landscape, in particular, supports intersubjective communication and argumentation. 


\section{$5 \quad$ Further Developments}

Conceptual information systems have been developed up to now based on the management system TOSCANA. They have been implemented for many purposes in different research areas, but also on the commercial level. Their range covers a variety of tasks and applications for which the human-centered view is dominant. For instance, TOSCANA information systems have been established for analyzing data of children with diabetes [21], for investigating international cooperations [11], for exploring laws and regulations in civil engineering [13], for retrieving books in a library [12], [19], for assisting engineers in designing pipings [27], for inquiring flight movements at Frankfurt Airport [10], for inspecting the control system of an incineration plant [9], for developing qualitative theories in music esthetics [15], for studying semantics of speech-act verbs [8], for examining the medical nomenclature system SNOMED [20], for controlling the customer database of a mail-order company [16] etc.

Further research aims at developing conceptual information systems by extending the functionalities that are available by the management system TOSCANA. Conceptual information systems may be understood as On-Line Analytical Processing (OLAP) tools [24]. The conceptual scales can roughly be seen as dimensions of a multi-dimensional data cube. The zooming in one of the concepts of a scale corresponds to 'slicing' the data cube. 'Dicing' and 'Drill-Down' are also supported. Another direction of research is concerned with extending the logic-based components. Since Formal Concept Analysis and Description Logics are closely related and have similar purposes (cf., e. g., [5], [25]), first steps in integrating both theories have been made ([1], [23], [3], [17]). Furthermore, the extension of Formal Concept Analysis to "Contextual Logic" (see [30], [18]) allows to integrate developments in the field of Conceptual Graphs. For hybrid information processing, an extension of TOSCANA information systems is projected by incorporating statistical and computational components [26]. All this indicates a promising development of extending TOSCANA information systems toward a wider range of applications.

\section{References}

1. F. Baader: Computing a minimal representation of the subsumption lattice of all conjunctions of concepts defined in a terminology. In: Proc. KRUSE'95, 168-178.

2. K. Becker, M. Zickwolff: IT-Sicherheitsmanagement mit TOSCANA. Intl. Conf. of the Information Systems Audit and Control Association, Brussels, July 13, 1998

3. H. Berg: Terminologische Begriffslogik. Diplomarbeit, FB4, TU Darmstadt 1997.

4. R. J. Brachman, T. Anand: The process of knowledge discovery in databases. In: U.M. Fayyad, G. Piatetsky-Shapiro, P. Smyth, R. Uthurusamy (eds.), Advances in Knowledge Discovery and Data Mining. Cambridge, Mass.: AAAI/MIT Press, 1996.

5. R. J. Brachman, P. G. Selfridge, L. G. Terveen, B. Altman, A. Borgida, F. Halper, T. Kirk, A. Lazar, D. L. McGuinnes, L. A. Resnick: Integrated support for data archaeology. International Journal of Intelligent and Cooperative Information Systems, Vol.2, No. 2, 1993, 159-185. 
6. Bundesamt für Sicherheit in der Informationstechnik: IT-Grundschutzhandbuch 1996. Bonn 1996.

7. B. Ganter, R. Wille: Formal Concept Analysis: Mathematical Foundations. Springer, Berlin-Heidelberg-New York 1999.

8. A. Grosskopf, G. Harras: Eine TOSCANA-Anwendung für Sprechaktverben des Deutschen. In: G. Stumme, R. Wille (eds.): Begriffiche Wissensverarbeitung: Methoden und Anwendungen. Springer, Berlin-Heidelberg-New York 2000, 273-295

9. E. Kalix: Entwicklung von Regelkonzepten für thermische Abfallbehandlungsanlagen. Diplomarbeit, FB13, TU Darmstadt, 1997.

10. U. Kaufmann: Begriffliche Analyse über Flugereignisse - Implementierung eines Erkundungs- und Analysesystems mit TOSCANA. Diplomarbeit, FB4, TU Darmstadt 1996.

11. B. Kohler-Koch, F. Vogt: Normen und regelgeleitete internationale Kooperationen. In: G. Stumme, R. Wille (eds.): Begriffliche Wissensverarbeitung: Methoden und Anwendungen. Springer, Berlin-Heidelberg-New York 2000, 325-340

12. W. Kollewe, C. Sander, R. Schmiede, R. Wille: TOSCANA als Instrument der der bibliothekarischen Sacherschließung. In: H. Havekost, H.J. Wätjen (eds.): Aufbau und Erschließung begrifflicher Datenbanken. (BIS)-Verlag, Oldenburg, 1995, 95114.

13. W. Kollewe, M. Skorsky, F. Vogt, R. Wille: TOSCANA - ein Werkzeug zur begrifflichen Analyse und Erkundung von Daten. In: R. Wille, M. Zickwolff (eds.): Begriffliche Wissensverarbeitung - Grundfragen und Aufgaben. B.I.-Wissenschaftsverlag, Mannheim, 1994, 267-288.

14. R. Langsdorf, M. Skorsky, R. Wille, A. Wolf: An approach to automated drawing of concept lattices. In: K. Denecke and O. Lüders (eds.): General Algebra and Applications in Discrete Mathematics. Shaker, Aachen 1997, 125-136.

15. K. Mackensen, U. Wille: Qualitative text analysis supported by conceptual data systems. Quality and Quantity: International journal of methodology 2/33 (1999), $135-156$

16. N. Mager: Formale Begriffsanalyse und Cluster-Analyse - ein Vergleich anhand der Identifikation von Artikel- und Auftragsstrukturen. Diplomarbeit, FB 1, TU Darmstadt 1998

17. S. Prediger: Logical scaling in formal concept analysis. In: D. Lukose, H. Delugach, M. Keeler, L. Searle, J. Sowa (eds.): Conceptual Structures: Fulfilling Peirce's Dream. Springer, Berlin-Heidelberg-New York 1997, 332-341.

18. S. Prediger: Kontextuelle Urteilslogik mit Begriffsgraphen. Ein Beitrag zur Restrukturierung der mathematischen Logik. Shaker Verlag 1998.

19. T. Rock, R. Wille: Ein TOSCANA-System zur Literatursuche. In: G. Stumme, R. Wille (eds.): Begriffliche Wissensverarbeitung: Methoden und Anwendungen. Springer, Berlin-Heidelberg 2000, 239-253

20. M. Roth-Hintz, M. Mieth, T. Wetter, S. Strahringer, B. Groh, R. Wille: Investgating SNOMED by Formal Concept Analysis. Preprint, FB4, TU Darmstadt 1997.

21. P. Scheich, M. Sorsky, F. Vogt, C. Wachter, R. Wille: Conceptual data systems. In: O. Opitz, B. Lausen, R. Klar (eds.): Information and classification. Springer, Berlin-Heidelberg 1993, 72-84.

22. H. Söll: Begriffliche Analyse triadischer Daten: Das IT-Grundschutzhandbuch des Bundesamts für Sicherheit in der Informationstechnik. Diplomarbeit, FB4, TU Darmstadt 1998.

23. G. Stumme: The conceptual classification of a terminology extended by conjunction and disjunction. In: N. Foo, R. Goebel (eds): Topics in Artificial Intelligence. Springer, Berlin-Heidelberg-New York 1996, 121-131. 
24. G. Stumme: On-Line Analytical Processing with Conceptual Information Systems. Proc. 5th Intl. Conf. on Foundations of Data Organization, 12.-11. November 1998

25. G. Stumme, R. Wille, U. Wille: Conceptual knowledge discovery in databases using formal concept analysis. In: J. M. Żytkow, M. Quafofou (eds.): Principles of Data Mining and Knowledge Discovery. Proc. of the 2nd European Symposium on PKDD '98, LNAI 1510, Springer, Heidelberg 1998, 450-458

26. G. Stumme, K. E. Wolff: Computing in conceptual data systems with relational structures. In: G. Mineau, A. Fall (eds.): Proceedings of the Second International Symposium on Knowledge Retrieval, Use, Storage for Efficiency. Simon Fraser University, Vancouver 1997, 206-219.

27. N. Vogel: Ein begriffliches Erkundungssystem für Rohrleitungen. Diplomarbeit, FB4, TU Darmstadt 1995.

28. F. Vogt, R. Wille: TOSCANA - a graphical tool for analyzing and exploring data. In: R. Tamassia, I.G. Tollis (eds.): Graph Drawing '94. LNCS 894. Springer, Berlin-Heidelberg-New York 1995, 226-233.

29. R. Wille: Begriffsdenken: Von der griechischen Philosophie bis zur Künstlichen Intelligenz heute. Dilthey-Kastanie, Ludwig-Georgs-Gymnasium, Darmstadt 1995, $77-109$

30. R. Wille: Conceptual graphs and formal concept analysis. In: D. Lukose, H. Delugach, M. Keeler, L. Searle, J. Sowa (eds.): Conceptual Structures: Fulfilling Peirce's Dream. Springer, Berlin-Heidelberg-New York 1997, 290-303.

31. R. Wille: Conceptual landscapes of knowledge: a pragmatic paradigm for knowledge processing. In: G. Mineau, A. Fall (eds.): Proceedings of the Second International Symposium on Knowledge Retrieval, Use, Storage for Efficiency. Simon Fraser University, Vancouver 1997, 2-13. 